\title{
Ivermectina dentro del protocolo de tratamiento para la COVID-19 en Perú: Uso sin evidencia científica
}

\author{
Ivermectin within the treatment protocol for COVID-19 in Peru: Use without scientific evidence
}

\author{
Jesús Lescano ${ }^{1}$, Chris Pinto ${ }^{2}$
}

RESUMEN

Ivermectina es uno de los fármacos antiparasitarios más ampliamente utilizados en medicina veterinaria. Asimismo, en medicina humana es utilizada en el tratamiento de diversas parasitosis. Sin embargo, su uso masivo sin control viene siendo asociado a la presentación de resistencia parasitaria. La emergencia del SARS-CoV-2, causante de la COVID-19, ha desafiado a los sistemas de salud a nivel mundial, forzándolos a trabajar urgentemente en el desarrollo de protocolos de tratamiento adecuados para dicha enfermedad. En el Perú, el Ministerio de Salud dispuso la inclusión de ivermectina dentro del protocolo de tratamiento contra la COVID-19. Con el objetivo de conocer la evidencia científica que respalda dicha medida, se realizó una búsqueda sistemática en repositorios digitales de revistas científicas y en las bases de datos de ensayos clínicos enfocados en tratamientos contra la COVID-19. Hasta la fecha de remisión de este artículo, no se encontró evidencia científica que respalde la eficacia ni seguridad del uso de ivermectina como tratamiento contra el SARS-CoV-2 en seres humanos. El único indicio es un estudio in vitro, cuya replicación en pacientes seria poco probable debido a que se necesitarían usar dosis considerablemente más altas que aquellas actualmente aprobadas para consumo humano. Adicionalmente, se encontró registro de algunos ensayos clínicos evaluando la inclusión de ivermectina en pacientes COVID-19, cuyos resultados aún no han sido publicados. Finalmente, este artículo describe tanto las consecuencias que podrían tener la desinformación generalizada respecto a ivermectina como tratamiento contra la COVID-19 sumada a la permisibilidad de que productos veterinarios conteniendo dicho principio activo sean empleados en pacientes humanos, así como, el importante rol de los Médicos Veterinarios en esta situación.

PALABRAS CLAVE: SARS-CoV-2, salud pública, medicina basada en la evidencia.

\section{SUMMARY}

Ivermectin is one of the most widely used antiparasitic drugs in veterinary medicine. Moreover, it is used for treating many parasitosis in human medicine. However, its uncontrolled massive use is being associated to the appearance of parasitic resistance. The emergence of SARS-CoV-2, causing agent of COVID-19, has challenged health systems worldwide, forcing them to urgently work in the development of proper therapeutic protocols against such disease. In Peru, the Ministry of Health approved the addition of ivermectin within the treatment protocol for COVID-19. Aiming to collect scientific evidence that supported such decision, a systematic search was performed within scientific journals' digital repositories and clinical trials' databases focused on therapy against COVID-19. Until the date of submission of this article, no scientific evidence was found to support the efficacy or safety of using ivermectin as treatment against SARS-CoV-2 in humans. Solely an in vitro study was found as suggestive evidence, but its replication in people would be unlikely as it would be necessary to administer considerably higher

Asociación de Médicos Veterinarios Voluntarios frente a Emergencias y Desastres del Perú. Lima, Perú.

London School of Hygiene and Tropical Medicine. Londres, Inglaterra. 
doses of the drug than those approved for use in humans. Additionally, some clinical trials are currently assessing the addition of ivermectin in COVID-19 patients, but their results have not been published yet. Finally, this article describes both the possible consequences of the generalized misinformation regarding the use of ivermectin against COVID-19 added to the permissibility for using veterinary products containing such drug in human patients, as well as the important role of veterinarians in this situation.

KEY WORDS: SARS-CoV-2, public health, evidence-based medicine.

\section{INTRODUCCIÓN}

Tras ser descubierta a mediados los años 1970, ivermectina fue la primera droga endectocida perteneciente al grupo de las lactonas macrocílicas; comercialmente disponible a inicios de 1980s y convirtiéndose en el producto de salud animal más vendido en el mundo para fines de la misma década (Prichard, 2007; Laing, Gillan y Devaney, 2017). En pacientes humanos, ivermectina es actualmente la única droga administrada oralmente para el tratamiento de sarna y también es empleada contra otras parasitosis como: pediculosis, oncocercosis, filariasis linfática y estrongiloidiasis (Prichard, 2007; Khalil, Abbas, Kibbi y Kurban, 2017; Patrì y Fabbrocini, 2020). Sin embargo, la seguridad de su administración a niños, gestantes y ancianos no ha sido probada y actualmente su uso masivo viene contribuyendo al incremento de la incidencia de resistencia parasitaria (Thomas et., 2015; Khalil et al., 2017). Con el pasar de los años, se han ido desarrollando otras lactonas macrocíclicas para uso en terapéutica veterinaria y control de plagas en cultivos (Prichard, 2007).

Recientemente, un estudio in vitro encontró que ivermectina inhibe la replicación del virus SARSCoV2 (causante de la COVID-19) y postula que el mecanismo de acción podría ser la inhibición de la importación nuclear de proteínas virales mediada por IMP $\alpha / \beta 1$, aunque se requieren estudios adicionales para corroborar dicha hipótesis (Caly, Druce, Catton, Jans y Wasstaff, 2020).

Por otra parte, es conocido que debido a la urgencia de disponer de información relacionada a la COVID-19 a nivel mundial, las principales editoriales científicas han admitido (y en algunos casos solicitado) que los artículos, relacionados a esta enfermedad, remitidos a evaluación para ser publicados sean simultáneamente archivados (en sus versiones no revisadas por pares ni aceptadas para publicación) en repositorios online de acceso abierto (ejm.: medRxiv, bioRxiv). Esta iniciativa ha sido considerada plausible por la comunidad científica; sin embargo, durante el proceso de revisión por pares se pueden detectar errores metodológicos o de análisis estadístico, cuya corrección puede implicar cambios considerables en la significancia de los resultados y el direccionamiento de las conclusiones. Por lo tanto, tal y como lo advierten los propios repositorios de artículos en proceso de revisión, la información vertida en éstos debe ser cautelosamente escudriñada antes de ser considerada correcta y es preferible esperar la versión "revisada por pares" o "aceptada para publicación" para ser empleada como referencia científica.

El 8 de mayo del 2020, mediante Resolución Ministerial No270-2020, el Ministerio de Salud del Perú (MINSA) aprueba la inclusión ivermectina (presentación: solución oral en gotas) dentro del protocolo de tratamiento para casos leves, moderados y severos de COVID-19 (Ministerio de Salud del Perú, 2020). Asimismo, dicho documento señala que el protocolo propuesto se basa en las recomendaciones recogidas del informe "Intervenciones Farmacológicas para el tratamiento de la enfermedad por el coronavirus 2019 (COVID-19)" (Instituto Nacional de Salud, 2020), el cual presenta una revisión rápida de la evidencia científica actualmente disponible sobre drogas que se han postulado como potencialmente efectivas en el tratamiento de casos de COVID-19. Este informe recopiló información procedente de un total de 15 estudios (6 reportes de caso, 6 series de casos y tres ensayos clínicos) publicados en el año 2020, donde se evaluaron los siguientes fármacos: Hidroxicloroquina, Favipiravir, Lopinavir/ritonavir, Umifenovir (arbidol o abidol), Lopinavir + interferon, Lopinavir + arbidol e interferón alfa.

Es importante resaltar que en el citado informe no se hace mención alguna sobre ivermectina y se concluye que hasta la fecha de su publicación no existe tratamiento farmacológico efectivo y seguro contra la COVID-19, tal y como también lo señalaron previamente Bendezú-Quispe, Rodríguez-Zúñiga, Román, Mori-Llontop, Peralta y Fiestas (2020). A pesar de esto, la RM No270-2020 no señala que el uso de ivermectina debe estar enmarcado en un ensayo 
clínico, con las características que le corresponden (ejm.: firma de consentimiento informado del paciente). Posteriormente, el 10 de mayo del 2020, el Colegio Médico Veterinario del Perú (CMVP) emite el Pronunciamiento titulado "Ivermectina en la salud de humanos, animales y ambiente". En éste se manifiesta expresamente el apoyo del CMVP a la medida propuesta por el MINSA sobre la inclusión de ivermectina dentro del protocolo de tratamiento para casos de COVID-19 y posteriormente se solicita la autorización para que ivermectina de uso veterinario pueda ser empleada en pacientes humanos.

El objetivo del presente trabajo fue investigar el sustento científico detrás de la inclusión de ivermectina dentro del protocolo de tratamiento contra la COVID-19, Para dicho fin se buscó literatura científica en las principales bases de datos digitales. Asimismo, se revisó la información emitida por la Administración de Alimentos y Medicamentos de los Estados Unidos (FDA) y Comunidad Europea respecto al uso de ivermectina en el tratamiento de la COVID-19. Adicionalmente, con el propósito de conocer los productos veterinarios conteniendo ivermectina registrados en Perú se consultó la base de datos del Sistema Integrado de Gestión de Insumos Agropecuarios (SIGIA) del Servicio Nacional de Sanidad Agraria (SENASA) de Perú. Finalmente, con el fin de conocer los ensayos clínicos evaluando el uso de ivermectina dentro del tratamiento contra la COVID-19, se realizaron búsquedas simultáneas en las bases de datos del Registro Peruano de Ensayos Clínicos, del Instituto Nacional de Salud de los Estados Unidos (NIH) y de la Organización Mundial de la Salud (OMS).

\section{METODOLOGÍA DE REVISIÓN DE LA LITERATURA}

El 20 de mayo del 2020 se realizó la revisión de los motores de búsqueda de artículos científicos digitales: PubMed, Medline, ScienceDirect, Scopus, Taylor \& Francis, SAGE y Wiley; empleando las palabras clave "ivermectin" AND "covid". En la misma fecha, empleando las palabras clave "FDA" AND "ivermectin" y "comisión europea" AND "ivermectina" en el motor de búsqueda Google, se recopiló información sobre la postura de la FDA y la Comunidad Europea sobre el uso de ivermectina contra la COVID-19. Posteriormente (27 de mayo de 2020), se realizó la búsqueda de productos veterinarios conteniendo el principio activo "ivermectina" en la base de datos SIGIA del SENASA (www.servicios.
senasa.gob.pe/SIGIAWeb/ip_productofarmaco.html). Finalmente, 20 de mayo del 2020, empleando las palabras clave "ivermectin" AND "covid", se revisaron las bases de datos del Registro Peruano de Ensayos Clínicos (www.ensayosclinicos-repec-ins.gob.pe), el Instituto Nacional de Salud de los Estados Unidos (www.clinicaltrials.gov) y la Organización Mundial de la Salud (http://www.apps-who.int/trialsearch).

\section{Acción antiviral antiviral in vitro de la ivermectina}

La evidencia actualmente disponible sobre el uso de ivermectina como potencial tratamiento de la COVID-19 no proviene resultados de ensayos clínicos. El estudio in vitro publicado por Caly et al. (2020) propone que ivermectina inhibe la replicación viral de SARS-CoV-2. Éste también indica que dicha droga fue previamente evaluada como tratamiento contra el virus del Dengue con resultados favorables in vitro que posteriormente no pudieron ser replicados in vivo; por lo tanto, concluye que se requieren estudios clínicos controlados en humanos para evaluar el uso efectivo y seguro de ivermectina como parte del tratamiento de COVID-19. Por su parte, Chaccour, Hammann, Ramón-García y Rabinobich (2020) señalan que la concentración plasmática de ivermectina alcanzada en humanos que recibieron 10 veces la dosis aprobada por laAdministración de Drogas y Alimentos de los Estados Unidos (FDA) es 10 veces inferior a la concentración considerada efectiva en el estudio in vitro de Caly et al. (2020). Además, los resultados de las simulaciones empleando modelos farmacocinéticos realizadas por Schmidt, Zhou y Lohmer (2020) sugieren que es poco probable obtener un ensayo clínico exitoso empleando las dosis actualmente aprobadas de ivermectina para humanos. Consecuentemente, la aplicación clínica de dicho fármaco es debatible. Adicionalmente, Chaccour et al. (2020) indican que ivermectina debe ser evaluada en ensayos clínicos controlados bajo extrema cautela debido a potenciales efectos adversos, tales como: a) neurotoxicidad en pacientes con incremento de la permeabilidad de la barrera hematoencefálica asociada a estado hiperinflamatorio y b) exposición incrementada a ivermectina en pacientes con disminución del citocromo $\mathrm{P}_{450} 3 \mathrm{~A} 4$ (principal ruta metabólica de esta droga) debido al uso concurrente de antiretrovirales (lopinavir/ritonavir y darunavir/cobicistat).

En contraparte, Patrì y Fabbrocini (2020) sugieren el potencial farmacológico de ivermectina para inhibir la replicación de virus RNA (como SARS-CoV-2) pero destacan que no se cuentan con estudios in vivo 
hasta la fecha. Finalmente, Wong (2020) advierte que la sobredosis de ivermectina puede conllevar a problemas gastrointestinales, hipersalivación, mareos, debilidad muscular, taquicardia, hipotensión, ataxia, agitación, rabdomiolisis y coma. Adicionalmente, algunos documentos vienen citando el estudio de Patel et al. (2020) como respaldo de la eficacia del uso de ivermectina en el tratamiento de la COVID-19. Sin embargo, cabe resaltar que dicho artículo no ha sido aún revisado por pares o formalmente publicado en ninguna revista (a la fecha 2 de junio del 2020), sino que únicamente se encuentra disponible en un repositorio online de acceso abierto. Además, Patel et al. (2020) señalan que sus resultados no deben ser considerados concluyentes sino preliminares. Finalmente, al revisar dicho manuscrito se observan discrepancias entre los resultados descritos y las figuras que los detallan, no se explica claramente si el "uso de ventilador mecánico" es un resultado o un factor de los grupos evaluados, etc.; lo cual no invalida el estudio, pero sí sugiere que éstos y otros detalles posiblemente serán corregidos durante el proceso de revisión por pares. Por lo tanto, se recomienda esperar la publicación formal del estudio antes de considerarlo como evidencia válida.

\section{Opinión de la FDA y Comunidad Europea sobre el uso de ivermectina en el tratamiento de la COVID-19}

Ivermectina no ha sido aprobado por la FDA para su uso como tratamiento de la COVID-19 (American Veterinary Medical Association, 2020). Aunque es cierto que dicho fármaco ha sido aprobado por la FDA para su uso en humanos en el tratamiento de parasitosis internas y externas, la misma entidad señala que las personas no deben consumir drogas de uso veterinario, ya que su seguridad y eficacia han sido evaluadas únicamente en las especies señaladas en la etiqueta y su uso en humanos podría causar daños severos (U.S. Food and Drug Administration, 2020).

Ivermectina de uso veterinario no ha sido aprobada por la Comunidad Europea para su uso en pacientes humanos. A través de la prensa (Mesquida, 2020) se ha distribuido la idea tergiversada que "ivermectina elimina el nuevo coronavirus en 48 horas"; $\sin$ especificar que la única evidencia corresponde a un estudio in vitro. El citado comunicado de la Comisión Europea textualmente señala: "En caso de escasez crítica de medicamentos esenciales autorizados para uso humano, también debe sopesarse la utilización de medicamentos equivalentes autorizados para uso veterinario que presenten los mismos principios activos, dosis y forma farmacéutica. La sustitución debe ser evaluada siempre meticulosamente $y$ autorizada por la autoridad nacional competente, teniendo en cuenta las posibles características especificas del sector veterinario" (Comisión Europea, 2020). Nótese que es una recomendación que debe evaluarse meticulosamente solo en una situación crítica y cumpliendo algunos requisitos. Además, no se hace mención alguna específicamente a ivermectina.

\section{Fármacos conteniendo ivermectina para uso animal}

La base de datos del Sistema Integrado de Gestión de Insumos Agropecuarios (SIGIA) del Servicio Nacional de Sanidad Agraria (SENASA) de Perú (www.servicios.senasa.gob.pe/SIGIAWeb/ ip_productofarmaco.html) muestra un total de 403 productos veterinarios conteniendo ivermectina con registro vigente, pertenecientes a 113 empresas titulares y en 17 formas farmacéuticas (gráfico 1). Entre éstas, las soluciones y suspensiones orales suman 53 (13.2\%); de las cuales, la mayoría (49) están formuladas en combinación con otros principios activos (ejm.: fenbendazol, praziquantel, pamoato de pirantel, etc.). Asimismo, las especies de destino son: perros, cerdos, equinos, caprinos, porcinos, bovinos, camélidos, cuyes, etc. Por lo tanto, el uso en pacientes humanos de estos productos podría presentar problemas de seguridad y eficacia; debido a: presentar formas farmacéuticas no recomendadas en humanos, estar combinados con otras drogas y estar diseñados para especies con características fisiológicas (ejm. $\mathrm{pH}$ gástrico, tasa metabólica, etc.) diferentes. Finalmente, la Dirección General de Medicamentos, Insumos y Drogas (2020b) ha señalado que los productos veterinarios conteniendo ivermectina no cumplen las exigencias (desde su formulación hasta su fabricación como producto final) de los medicamentos para uso humano; por lo tanto, su uso en pacientes humanos podría causar reacciones adversas, toxicidad o ineficacia terapéutica.

\section{Estudios clínicos sobre el uso de ivermectina contra la COVID-19}

A la fecha de búsqueda, se encontró estudio alguno sobre el uso de ivermectina como tratamiento para la COVID-19 en la base de datos del Registro Peruano de Ensayos Clínicos. Por su parte, las bases de datos de ensayos clínicos del Instituto Nacional de Salud de los Estados Unidos (www.clinicaltrials.gov) y de la Organización Mundial de la Salud (http://www.appswho.int/trialsearch) señalan registrados un total de 


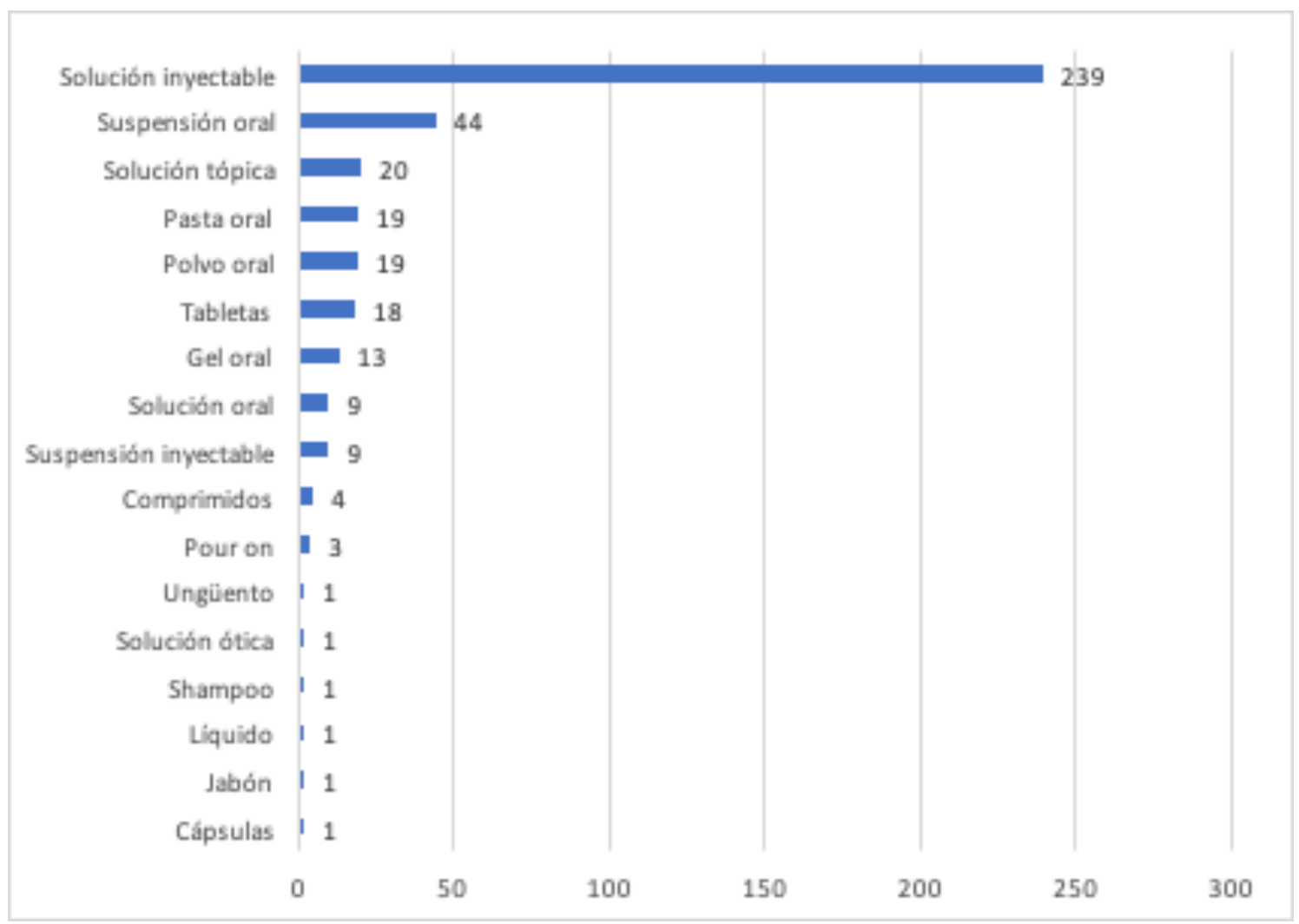

Gráfico 1. Número de productos veterinarios con registro vigente conteniendo ivermectina (Fuente: SIGIA).

19 estudios (6 de éstos en fase de reclutamiento) que incluyen a ivermectina (sola o junto a otros fármacos) y ninguno de éstos con sede en Perú. Al respecto, la revista Nature (2020) señala que actualmente hay un exceso de ensayos clínicos, con tamaños muestrales pequeños, sin incluir un grupo control y sin significancia estadística, en búsqueda de tratamientos efectivos contra la COVID-19. Consecuentemente, los resultados obtenidos por éstos no son robustos o son posteriormente invalidados ya sea durante el proceso de revisión por pares o por estudios mejor estructurados. Por lo tanto, se recomienda desarrollar estudios colaborativos que involucren equipos de diversos países trabajando en simultáneo, como el estudio Solidaridad llevado a cabo actualmente por la Organización Mundial de la Salud.

\section{Desinformación induce a la automedicación en la población}

Lo anteriormente expuesto discrepa de la información que se viene distribuyendo a la población peruana a través de los medios de comunicación (Caretas, 2020) y resalta la necesidad de tener evidencia científica que respalde o descarte a cualquier droga que sea propuesta como efectiva en el tratamiento de la COVID-19. Un ejemplo de fármacos aparentemente promisorios y ampliamente empleados en el tratamiento de la COVID-19 son cloroquina e hidroxicloroquina; cuyo beneficio no pudo ser confirmado, pero sí se asoció a incremento en la tasa de mortalidad, en un estudio que incluyó aproximadamente 96 mil pacientes de 671 hospitales de diversos países (Mehra, Desai, Ruschizka y Patel, 2020). Cabe resaltar que la desinformación generalizada respecto a ivermectina como tratamiento efectivo contra la COVID-19 sumada a la permisibilidad de que productos veterinarios conteniendo dicho principio activo sean empleados en pacientes humanos podría resultar en: 1) la compra indiscriminada de ivermectina de uso veterinario por parte de la población, 2) su administración en humanos sin prescripción ni supervisión médica, 3) presentación de morbilidad en las personas asociada a efectos adversos o sobredosis, como actualmente ya viene aparentemente reportando (Dirección General de Medicamentos, Insumos y Drogas, 2020a), 4) escasez del principio activo en su presentación para uso veterinario, 5) especulación e incremento de precios en el mercado farmacéutico (humano y veterinario), 6) expendio de productos adulterados y sin registro 
sanitario 7) incremento de la incidencia de resistencia frente al principio activo en casos de parasitosis en humanos, 8) incremento de la práctica empírica de la medicina veterinaria y posible intrusismo profesional en el campo de la medicina humana, entre otras consecuencias.

\section{CONCLUSIONES Y RECOMENDACIONES}

Considerando que, durante nuestra formación profesional, los Médicos Veterinarios adquirimos conocimientos sobre farmacología, bioestadística, epidemiología y otras ciencias, estamos capacitados para evaluar críticamente la evidencia científica que aparece en el campo de la biomedicina. Por lo tanto, ante la situación actual respecto al uso de ivermectina como parte del tratamiento de casos de COVID-19 y con la intención tanto de evitar el uso inapropiado de dicha droga en humanos como su posible escasez en el futuro próximo, los Médicos Veterinarios estamos llamados a cumplir los siguientes roles:

Emplear los canales e instancias correspondientes para emitir opinión basada en la evidencia respecto a las políticas estatales que involucren nuestras competencias. En este caso, hacer un llamado al Ministerio de Salud y al Colegio Médico del Perú, señalando que la decisión de usar de ivermectina como parte del protocolo de tratamiento contra COVID-19 solo se respalda por un estudio in vitro, por lo tanto, su uso en pacientes COVID-19 debe realizarse únicamente a través de estudios que sigan las directrices presentadas en el Decreto Supremo $\mathrm{N}^{\circ}$ 014-2020 S.A. que regula los ensayos clínicos de dicha enfermedad.

Socialmente y desde los diversos ámbitos de ejercicio profesional, los Médicos Veterinarios debemos cumplir el rol de compartir nuestro conocimiento y educar a la población, evitando que la desinformación se disemine y tenga consecuencias negativas para la salud humana, animal y ambiental. En este caso, advertir a la comunidad sobre los efectos adversos asociados al uso de medicamentos veterinarios en pacientes humanos. Adicionalmente, solicitar al CMVP un pronunciamiento exhortando a sus agremiados a no realizar recomendaciones sobre la administración de ivermectina (u otros medicamentos) de uso veterinario a pacientes humanos. Pues dicho acto constituiría, ante todo, intrusismo profesional de parte de los Médicos Veterinarios hacia el campo de la Medicina Humana, además de incrementar la confusión de la población y potencialmente conllevar a poner en riesgo la salud pública.

Controlar que el expendio de medicamentos de uso veterinario que contengan el principio activo ivermectina se limite a personas que presenten una receta firmada y sellada por un Médico Veterinario colegiado. Prestando especial atención (pero no limitándose) a aquellos productos en presentación suspensión o solución oral que únicamente contengan ivermectina como principio activo (Promectine oral ${ }^{\circ}$, Anglomec-S ${ }$, Iverdrog, S.O. ${ }^{\circledR}$ e Iversol $\left.{ }^{\circledR}\right)$ y que consecuentemente pudieran ser irresponsablemente empleados como reemplazo del medicamento de uso humano. Además, verificar que en ésta se especifique la dosis, forma de presentación, cantidad, frecuencia de administración y días de tratamiento, a fin de verificar que realmente corresponde a un protocolo real y apropiado para la especie indicada en la receta. Finalmente, retener y eliminar la receta cuando se haya vendido el medicamento.

En la medida de lo posible, evitar temporalmente recetar ivermectina dentro de los esquemas de tratamientos veterinarios; reemplazándola por otras drogas que puedan producir similar efecto terapéutico y que sea apropiada para la especie animal involucrada. En caso de ser estrictamente necesario el uso de ivermectina, restringirlo a su administración directa por el Médico Veterinario tratante y no por el propietario o cliente.

Solicitar al Servicio Nacional de Sanidad Agraria (SENASA) el diseño urgente de la normativa necesaria para la regulación y fiscalización del expendio de medicamentos de uso veterinario en tiendas distribuidoras, agro-veterinarias y otras similares. Asimismo, se recomienda re-categorizar a los productos conteniendo el principio activo ivermectina al Grupo I (Producto veterinario que se expende Bajo receta de Prescripción Restringida, expedida por un Médico Veterinario, la cual debe ser archivada por el establecimiento que realiza la venta; según RD-00882016-MINAGRI-SENASA-DIAIA) en el Clasificador de productos veterinarios y alimentos para animales vigente (disponible en: https://www.senasa.gob.pe/ senasa/descargasarchivos/2014/12/CLASIFICADORD E - P R O D U C T O S - V E T E R IN A R I O S - Y ALIMENTOS-PARA-ANIMALES.pdf).

Solicitar al Colegio Médico Veterinario del Perú (y sus dependencias Departamentales) vigilar y atender posibles casos de: práctica empírica de la medicina veterinaria (adquisición, expendio y administración de 
ivermectina de uso veterinario), intrusismo profesional en el campo de la Medicina Humana (tratamiento de pacientes humanos por parte de Médicos Veterinarios), adulteración y comercialización de productos veterinarios conteniendo ivermectina (con énfasis en los productos Promectine oral ${ }^{\circledR}$, Anglomec-S $\AA$, Iverdrog, S.O. ${ }^{\circledR}$ e Iversol ${ }^{\circledR}$, pues su presentación es similar a la recomendada para uso humano), incremento irregular en los volúmenes de venta de productos veterinarios conteniendo ivermectina (solicitar información a laboratorios y distribuidoras), entre otros.

\section{Correspondencia:}

Jesús Lescano

Correo electrónico: jlescanogomez@gmail.com

\section{REFERENCIAS BIBLIOGRÁFICAS}

1. American Veterinary Medical Association. (2020). FDA: Ivermectin not approved for COVI-19 treatment. Washington DC: American Veterinary Medical Association. Recuperado: https://www. avma.org/javma-news/2020-06-01/fda-ivermectinnot-approved-covid-19-treatment

2. Bendezú-Quispe, G., Rodríguez-Zúñiga, M., Román, Y., Mori-Llontop, L., Peralta, V., \& Fiestas, F. (2020). Agentes potencialmente terapéuticos contra el SARSCoV-2: Revisión rápida de la evidencia. Revista Peruana de Medicina Experimental y Salud Pública, 37 (2): 320-326.

3. Caly, L., Druce, J., Catton, M., Jans, D., \& Wasstaff, D. (2020). The FDA approved Drug Ivermectin inhibits the replication of SARS-CoV-2 in vitro. Antiviral Research, 178, 104787

4. Caretas. (2020). Una gran esperanza. Lima: Caretas. Rcuperado de: https://caretas.pe/caretas-en-casa/unagran-esperanza/

5. Chaccour, C., Hammann, F., Ramón-García, S., \& Rabinobich, N. (2020). Ivermectin and COVID-19: Keeping Rigor in Times of Urgency. Am J Trop Med Hyg, 102(6), 1156-1157.

6. Comisión Europea. (2020). Directrices para el suministro óptimo y racional de medicamentos a fin de evitar la escasez durante el brote de COVID-19. Paris: Diario Oficial de la Unión Europea Número 110. Recuperado de: https://eur-lex.europa.eu/ legal-content/ES/TXT/?uri=CELEX\%3 A52020 XC0408\%2803\%29

7. Dirección General de Medicamentos, Insumos y Drogas. (2020a). Ivermectina: recomendaciones de uso asociadas a infección por COVID-19. Alerta DIGEMID No ${ }^{\circ}$ 2. Lima: Dirección General de Medicamentos, Insumos y Drogas. Disponible en:
http://www.digemid.minsa.gob.pe/UpLoad/ UpLoaded/PDF/Alertas/2020/ALERTA_12-20.pdf

8. Dirección General de Medicamentos, Insumos y Drogas. (2020b). Uso indebido de ivermectina para animales en el tratamiento de COVID-19 en humanos. Alerta DIGEMID N ${ }^{\circ} 15$. Lima: Dirección General de Medicamentos, Insumos y Drogas. Recuperado de: http://www.digemid.minsa.gob.pe/UpLoad/ UpLoaded/PDF/Alertas/2020/ALERTA_15-20.pdf

9. Unidad de Análisis y Generación de Evidencias en Salud Pública / Instituto Nacional de Salud. (2020). Tratamiento especifico para la infección por COVID-19. Lima: Unidad de Análisis y Generación de Evidencias en Salud Pública/ Instituto Nacional de Salud.

10. Khalil, S., Abbas, O., Kibbi, A. \& Kurban, M. (2017). Scabies in the age of increasing drug resistance. PLoS Neglected Tropical Diseases 11(11): e0005920. DOI: https://doi.org/10.1371/journal.pntd.000592

11. Laing, R., Gillan, V. \& Devaey, E. (2017). Ivermectin - old drug, new tricks? Trends in Parasitology, 33(6):463-472. DOI:10.1016/j.pt.2017.02.004

12. Mehra, M., Desai, S., Ruschitzka, F., \& Patel, A. (2020). Hydroxychloroquine or chloroquine with or without a macrolide for treatment of COVID-19: a multinational registry analysis. The Lancet (retracted). DOI: doi.org/10.1016/S0140-6736(20) 31180-6

13. Mesquida, F. (2020). Ivermectina: la Unión Europea aprobó el uso de medicamentos veterinarios en humanos. Buenos Aires: INFOCAMPO. Recuperado de: https://www.infocampo.com.ar/ivermectinala-union-europea-aprobo-el-uso-de-medicamentosveterinarios-en-humanos/?fbclid=IwAR31YHf7 J ypyTOcBgZzeuT6dgT0oYXLkeAsIoJPMfDEC4O_ fqeea6KPpnOg

14. Ministerio de Salud del Perú. (2020). Resolución Ministerial No270-2020.Lima: Ministerio de Salud del Perú.

15. Nature. (2020). Editorials: Coronavirus drugs trials need scale and collaboration. Nature, 581, 120 doi: 10.1038/d41586-020-01391-9

16. Patel, A., Desai, S., Grainger, D. (2020). Usefulness of ivermectin in COVID-19 illness (En Revisión), SSRN.

17. Patrì, A., Fabbrocini, G. (2020). Hydroxychloroquine and ivermectin: A synergistic combination for COVID-19 chemoprophylaxis and treatment? Journal of the American Academy of Dermatology, e221

18. Prichard, R. (2007). Ivermectin resistance and overview of the Consortium for Antihelmintic Resistance SNPs. Expert Opinion on Drug Discovery, 2(1);S41-S52 DOI: 10.1517/17460441.2.S1.S41

19. Schmidt, V., Zhou, J. \& Lohmer, L. (2020). The approved dose of ivermectin alone is nor the ideal 
dose for the treatment of COVID-19. Clin Pharmacol Ther. DOI: $10.1002 /$ cpt.1889

20. Thomas, J., Peterson, G., Walton, S., Carson, C., Naunton, M. \& Baby, K. (2015). Scabies: an ancient global disease with a need for new therapies. BMC Infectious Diseases, 15, 250. DOI: https://doi. org/10.1186/s12879-015-0983-z

21. U.S. Food and Drug Administration. (2020). FAQ: COVID-19 and ivermectin intended for animals.
Washington DC: U.S. Food and Drug Administration. Recuperado de: https://www.fda.gov/animalveterinary/product-safety-information/faq-covid-19and-ivermectin-intended-animals

22. Wong, A. (2020). COVID-19 and toxicity from potential treatments: Panacea or poison. Emerg Med Australas,32(4),697-699. doi: 10.1111/17426723.13537 